\title{
Sevoflurane inhibits cell proliferation and migration of glioma by targeting the miR-27b/VEGF axis
}

\author{
XI ZHAN, CHANGCHENG LEI and LINZHU YANG \\ Department of Anesthesiology, The First Affiliated Hospital of Kunming Medical University, \\ Kunming, Yunnan 650032, P.R. China
}

Received October 5, 2018; Accepted February 26, 2020

DOI: $10.3892 / \mathrm{mmr} .2021 .12047$

\begin{abstract}
Poor prognosis in patients with glioma is primarily due to rapid tumor growth and cell invasion and migration. In addition, microRNA (miR)-27b is decreased in metastatic glioma. The present study investigated whether sevoflurane inhibited glioma cell progression by targeting miR-27b. Cell proliferation was analyzed using a Cell Counting Kit- 8 assay and a wound healing assay was used to detect cell migration. Western blotting and reverse transcription-quantitative PCR analysis were performed to determine the protein and mRNA expression levels. A dual luciferase assay was used to determine the relationship between vascular epithelial growth factor (VEGF) and miR-27b. VEGF was identified to be a direct target of miR-27b. Moreover, sevoflurane treatment increased the expression of miR-27b and decreased the expression of VEGF in U251 and U87 cells. Compared with the control group, sevoflurane inhibited the proliferation and migration of U251 and U87 cells, as well as the expression of matrix metalloproteinase (MMP)-2 and MMP-9, which were subsequently abolished by pre-treatment with an miR-27b inhibitor. The present results indicated the potential use of sevoflurane by anesthesiologists for the surgical resection of glioma, which may improve patient outcomes in the clinical setting.
\end{abstract}

\section{Introduction}

Glioma tumors originate from the glial cells of the brain or spine (1), with various oncogenes serving a role in its development (2). Certain angiogenic blockers, including bevacizumab, have been used in combination with conventional chemotherapy for the successful treatment of recurrent high-grade glioma (3).

A previous meta-analysis also compared the clinical outcomes of surgical resection and biopsy, as these are the first surgical interventions for patients with low-grade glioma (4).

Correspondence to: Dr Linzhu Yang, Department of Anesthesiology, The First Affiliated Hospital of Kunming Medical University, 295 Xichang Road, Kunming, Yunnan 650032, P.R. China E-mail: yanglinzhukmmu@sina.com

Key words: sevoflurane, cell proliferation, cell migration, glioma
Another meta-analysis compared radiotherapy against radiotherapy in combination with chemotherapy for the treatment of patients with high-grade glioma. The results of this analysis revealed that the latter strategy demonstrated a small but clear improvement in the outcomes of treatments (5). While there are several treatment options for patients, including surgery, radiation and chemotherapy, the median overall survival of patients with malignant glioma is 1-2 years (6). Moreover, the poor prognosis of patients with glioma is largely a result of rapid tumor growth and cell invasion and migration (7). Therefore, suppressing cell proliferation and migration may serve as a novel therapeutic strategy for patients with glioma.

Anesthetics and anesthesia techniques impact the migration of tumor cells (8). Sevoflurane, a volatile anesthetic agent, inhibits the proliferation of colonic (9) and laryngeal cancer cells (10), and inhibits the migration of lung cancer cells (11). Furthermore, sevoflurane has been reported to modulate multiple microRNAs (miRNAs/miRs) in the brain (12). However, the effect of sevoflurane on the migration and proliferation of glioma cells is unknown.

miRNAs are a group of small, non-coding RNAs comprised of 21-23 nucleotides (13). miRNAs regulate gene expression by binding to the 3'-untranslated regions of target mRNAs (14). Moreover, miRNAs serve crucial roles in multiple oncogenic activities, including proliferation, migration, invasion and angiogenesis (15). In addition, miRNAs are deregulated in various types of cancer, including glioblastoma (16). miR-27b acts as an important tumor suppressor in numerous cancer types and reduces tumor growth and metastasis via targeting nuclear receptor subfamily 2 group $\mathrm{F}$ member 2 in gastric cancer (17). Moreover, miR-27b induces cell apoptosis and reduces cell viability and survival by targeting frizzled class receptor 7 in lung cancer (18), and reduces cell growth and invasion via targeting Rab3D in colorectal cancer (19). In addition, miR-27b also serves a tumor suppressor in glioma and miR-27b expression is significantly lower in metastatic glioma tissues compared with non-metastatic tissues (20). However, whether sevoflurane regulates glioma cell migration and proliferation by targeting miR-27b is yet to be elucidated.

\section{Materials and methods}

Cell lines. Normal human glial HEB cells and U251 (U251-MG; has not been authenticated yet; glioma cells; 
Type culture Collection of the Chinese Academy of Science) and U87 (cat. no. ATCC ${ }^{\circledR} \mathrm{HTB}-14$; has not been authenticated yet; glioblastoma of unknown origin; American Type Culture Collection) glioma cell lines were cultured in DMEM (Invitrogen; Thermo Fisher Scientific, Inc.) supplemented with 10\% FBS (Invitrogen; Thermo Fisher Scientific, Inc.), 1\% penicillin and $1 \%$ streptomycin at $37^{\circ} \mathrm{C}$ in $5 \% \mathrm{CO}_{2}$.

miRNA transfection. miR-27b mimics (5'-CGTCTTGAA TCGGTGACACTT-3'), miR-27b inhibitors (5'-GGUAAU CCCUGGCAAUGUGAU-3') and miR-negative control (miR-NC; 5'-UUGUACUACACAAAAGUACUG-3') were purchased from Shanghai GenePharma Co., Ltd. Once cells reached a confluence of $50-70 \%$, they were transfected with the aforementioned agents using Lipofectamine ${ }^{\circledR} 2000$ reagent (Invitrogen; Thermo Fisher Scientific, Inc.) in accordance with the manufacturer's protocol. The final working concentration of the miR-27b mimic, miR-27b inhibitor or miR-NC was $40 \mathrm{nmol} / \mathrm{l}$. Following transfection for $24 \mathrm{~h}$ at $37^{\circ} \mathrm{C}$, the cells were collected for subsequent experimentation.

Small interfering (si)RNA transfection. Transfection with siRNA-NC $(0.01 \mu \mathrm{M} ; 5$ '-ACGUGACACGUUCGGAGA ATT-3'; Shanghai GenePharma Co., Ltd.) or siRNA vascular epithelial growth factor (VEGF; $0.01 \mu \mathrm{M}$; siRNA-1 VEGF, 5'-GAUCUCAUCAGG GUACUCCdTdT-3'; siRNA-2 VEGF, 5'-GTGCTGGCCTTGGTGAGGTTT-3'; Shanghai GenePharma Co., Ltd.) was performed using the TurboFect siRNA transfection reagent (Fermentas; Thermo Fisher Scientific, Inc.) in accordance with the manufacturer's protocol. At 48 h post-transfection, U251 and U87 cells were collected and used for further analyses.

Groups. U251 and U87 cells (1x10 5 cells/well) in the exponential growth phase were seeded into 6-well plates and incubated at $37^{\circ} \mathrm{C}$ overnight in DMEM (Thermo Fisher Scientific, Inc.). Cell exposure to $3.4 \%$ sevoflurane (Maruishi Pharmaceutical Co., Ltd.) for $6 \mathrm{~h}$ at $37^{\circ} \mathrm{C}$ was conducted according to a previous report (11). Cell plates were put into an airtight chamber that was connected to an anesthesia machine (Cicero-EM 8060; Drägerwerk AG \& Co. KGaA), attached to which was an anesthetic vaporizer (Sevorane; Abbott Pharmaceutical Co., Ltd.) that supplied sevoflurane and the sevoflurane concentration (3.4\%) was monitored using PM 8060 (Dräger KGaA). Subsequently, cells were randomly divided into the following four groups: Untreated control (95\% air and 5\% $\left.\mathrm{CO}_{2}\right)$, sevoflurane $\left(3.4 \%\right.$ sevoflurane mixed with $95 \%$ air and $5 \% \mathrm{CO}_{2}$ ), sevoflurane + miR-27b inhibitor (administered $48 \mathrm{~h}$ prior to sevoflurane) and sevoflurane + VEGF siRNA + miR-27b inhibitor. The untreated control group acted as a negative control group for the experiments. After cells were exposed to sevoflurane for $6 \mathrm{~h}$ at $37^{\circ} \mathrm{C}$, cells were grown at $37^{\circ} \mathrm{C}$ in a $5 \% \mathrm{CO}_{2}$ incubator for an additional $24 \mathrm{~h}$. Cells were then used for cell proliferation assays, migration assays or molecular analyses.

$R N A$ extraction and reverse transcription-quantitative ( $R T-q)$ $P C R$. Total miRNA from U251 and U87 cells was extracted using the mirVana miRNA Isolation kit (Ambion; Thermo Fisher Scientific, Inc.) in accordance with the manufacturer's protocol. Subsequently, cDNA was synthesized from $5 \mathrm{ng}$ total RNA using the TaqMan miRNA RT kit (Applied Biosystems; Thermo Fisher Scientific, Inc.) in accordance with the manufacturer's protocol. The following RT protocol was used: $37^{\circ} \mathrm{C}$ for $45 \mathrm{~min}$ and $65^{\circ} \mathrm{C}$ for $10 \mathrm{~min}$. The expression of miR-27b was quantified using the miRNA-specific TaqMan miRNA Assay kit (Applied Biosystems; Thermo Fisher Scientific, Inc.) with the Applied Biosystems 7500 RT PCR system (Applied Biosystems; Thermo Fisher Scientific, Inc.). The following thermocycling conditions were used for the qPCR: $95^{\circ} \mathrm{C}$ for $10 \mathrm{~min}$; followed by 40 cycles of $95^{\circ} \mathrm{C}$ for $10 \mathrm{sec}$ and $60^{\circ} \mathrm{C}$ for $1 \mathrm{~min}$. The relative quantification of $\mathrm{miR}-27 \mathrm{~b}$ was normalized to that of U6. The primer sequences were as listed: miR-27b forward, 5'-CGGCGGTTCACAGTGGCTAA-3' and reverse, 5'- GTGCAGGGTCCGAGGT-3'; and U6 forward, 5'-GCT TCGGCAGCACATATACTAAAAT-3' and reverse, 5'-CGC TTCACGAATTTGCGTGTCAT-3'.

Total RNA was isolated from U251 and U87 cells using TRIzol $^{\circledR}$ reagent (Invitrogen; Thermo Fisher Scientific, Inc.). cDNA was then reverse transcribed from total RNA using a SuperScript II RT kit (Invitrogen; Thermo Fisher Scientific, Inc.). The following RT temperature protocol was used: $50^{\circ} \mathrm{C}$ for $10 \mathrm{~min}$ and $80^{\circ} \mathrm{C}$ for $10 \mathrm{~min}$. qPCR was performed using the SYBR Green PCR Master Mix (Thermo Fisher Scientific, Inc.) and the Applied Biosystems 7500 RT PCR System (Applied Biosystems; Thermo Fisher Scientific, Inc.). The following thermocycling conditions were used for the qPCR: $95^{\circ} \mathrm{C}$ for $30 \mathrm{sec}$; followed by 40 cycles of $95^{\circ} \mathrm{C}$ for $5 \mathrm{sec}$ and $60^{\circ} \mathrm{C}$ for $30 \mathrm{sec}$. The expression of target genes was normalized to that of GAPDH. The primer sequences were as listed: Matrix metalloproteinase (MMP)-2 forward, 5'-GCCCCA GACAGGTGATCTTG-3' and reverse, 5'-GCTTGCGAG GGAAGAAGTTGT-3'; MMP-9 forward, 5'-AGACGGGTA TCCCTTCGACG-3' and reverse, 5'-AAACCGAGTTGG AACCACGAC-3'; and GAPDH forward, 5'-GGTCTCCTC TGACTTCAACA-3' and reverse, 5'-GTGAGGGTCTCTCTC TTCCT-3'. Expression levels were quantified using the $2^{-\Delta \Delta C q}$ method (21).

Cell proliferation assay. The proliferation of U251 and U87 cells was analyzed using a Cell Counting Kit-8 (CCK-8) assay (Beyotime Institute of Biotechnology), according to the manufacturer's protocol. In total, $3 \times 10^{3}$ cells $(100 \mu \mathrm{l})$ were seeded in 96-well plates and incubated for 24 and $48 \mathrm{~h}$. Subsequently, $10 \mu \mathrm{l} \mathrm{CCK}-8$ solution was added into each well and incubated for a further $1 \mathrm{~h}$ at $37^{\circ} \mathrm{C}$. Absorbance at $450 \mathrm{~nm}$ was then measured using an ELX-800 spectrometer reader (BioTek Instruments, Inc.).

Wound healing assays. U251 and U87 cells (1x105 cells/well) were seeded into 6-well plates and cultured to $100 \%$ confluence, after which a wound was created by manually scraping the cell monolayer with a $10 \mu \mathrm{l}$ pipette tip. Cells were washed with serum free medium to remove floating cells and then incubated in DMEM supplemented with $1 \% \mathrm{FBS}$ at $37^{\circ} \mathrm{C}$. Cell migration into the wound was observed at two time points ( 0 and $24 \mathrm{~h}$ ) in six randomly selected fields of view. Images were acquired using a phase-contrast Leitz light microscope (magnification, x100). The migratory distance of U251 and U87 cells was determined by subtracting the wound width at 
$24 \mathrm{~h}$ from the wound width at $0 \mathrm{~h}$ and analyzed using Image J software (version 1.8.0; National Institutes of Health). The obtained values were expressed as migration percentages, setting the gap width at $0 \mathrm{~h}$ as $0 \%$.

Western blot analysis. Total protein was extracted from cells using RIPA lysis buffer (Beyotime Institute of Biotechnology), according to the manufacturer's protocol. Total protein was quantified using a bicinchoninic acid assay kit (Beyotime Institute of Biotechnology). Subsequently, $15 \mu \mathrm{g}$ protein/lane was separated using 8-10\% SDS-PAGE and transferred onto a PVDF membrane, which was subsequently blocked with $5 \%$ skimmed milk diluted with TBS-1\% Tween (TBST) for $2 \mathrm{~h}$ at room temperature. The PVDF membrane was then incubated overnight at $4{ }^{\circ} \mathrm{C}$ with polyclonal antibodies for MMP-2 (cat. no. ab215986; 1:1,000; Abcam) and MMP-9 (cat. no. ab219372; 1:1,000; Abcam), with GAPDH antibody (cat. no. ab8245; 1:10,000; Abcam) serving as an internal control. The membrane was further incubated with horseradish peroxidase-conjugated anti-rabbit (cat. no. sc-2030) or anti-mouse (cat. no. sc-2005) immunoglobulin-G secondary antibodies (1:5,000; Santa Cruz Biotechnology, Inc.) diluted with TBST for $1 \mathrm{~h}$ at room temperature. The resulting protein signals were detected using an enhanced chemiluminescence reaction system (EMD Millipore) and quantified using ImageJ software (version 1.8.0; National Institutes of Health).

Luciferase assay. TargetScan release 7.1 (http://www. targetscan.org/vert_71/) was used in the present study for the prediction of binding site between miR-27b and VEGF. The 3'UTR of VEGF mRNA was amplified from the cDNA of HEB cells and inserted into a pGL3-basic plasmid (Promega Corporation). The pGL3-VEGF 3'UTR-mutant (Mut) was created by introducing VEGF 3'UTR site mutations using a Quick Site-Directed Mutation kit (Agilent Technologies, Inc.). U251 and U87 cells were co-transfected with miR-27b mimics (20 nM) or miR-NC (20 nM), pGL3-VEGF 3'UTR-wild type (WT; $0.4 \mathrm{mg}$ ) or pGL3-VEGF 3'UTR-Mut (0.4 mg) and Renilla luciferase vectors using Lipofectamine ${ }^{\circledR} 2000$ reagent (Invitrogen; Thermo Fisher Scientific, Inc.), according to the manufacturer's protocol. A dual luciferase assay was performed at $48 \mathrm{~h}$ after transfection using a Dual Luciferase kit (Promega Corporation). Activities were normalized to that of Renilla luciferase.

Statistical analysis. Each experiment was performed three times and data were analyzed using SPSS 16.0 software (IBM Corp.). Differences between two groups were analyzed using a two-tailed Student's t-test, while differences among $\geq 3$ groups were evaluated using one-way ANOVA followed by a Tukey's multiple comparison post hoc test. Data are presented as the mean \pm SEM. $\mathrm{P}<0.05$ was considered to indicate a statistically significant difference.

\section{Results}

Sevoflurane induces the expression of miR-27b in glioma cells. To determine the expression of miR-27b in glioma cells compared with normal cells, RT-qPCR was performed. It was found that U251 cells had significantly decreased miR-27b expression compared with HEB cells (Fig. 1A). RT-qPCR was also performed to determine whether sevoflurane effects the expression of miR-27b in glioma cells. It was demonstrated that U251 cells treated with sevoflurane had significantly increased miR-27b expression compared with the control group (Fig. 1B). Furthermore, in U87 cells, a decreased expression of miR-27b was identified compared with HEB cells (Fig. 1C). In addition, sevoflurane treatment also significantly increased miR-27b expression compared with the control group (Fig. 1D).

miR-27b mimics and inhibitors affect the expression of $m i R-27 b$. Following transfection with the miR-27b mimic, miR-27b was significantly increased in U251 cells (Fig. 2A). Moreover, after transfection with miR-27b inhibitors, miR-27b expression was significantly decreased in U251 cells (Fig. 2B). The same effects were observed in U87 cells (Fig. 2C and D).

VEGF is a target of $m i R-27 b$. TargetScan was used to search for the potential targets of miR-27b in humans and a conserved binding site for miR-27b in the 3'UTR region of the VEGF gene was identified (Fig. 3A).

To further assess whether miR-27b alters the expression of VEGF by post-transcriptionally regulating its 3'UTR, a luciferase reporter plasmid containing the 3'UTR of VEGF was constructed. It was found that the luciferase activity of miR-27b mimic + pGL3-VEGF 3'UTR-WT-transfected U251 and U87 cells was significantly decreased compared with miR-NC + pGL3-VEGF 3'UTR-WT-transfected cells (Fig. 3B and C).

Sevoflurane reduces the expression of VEGF in glioma cells by targeting $m i R-27 b$. To determine the expression of VEGF in glioma cells compared with normal cells, VEGF expression was determined via RT-qPCR. It was found that U251 cells had significantly increased expression levels of VEGF compared with HEB cells (Fig. 4A).

To examine whether sevoflurane effects the expression of VEGF in glioma cells, RT-qPCR was performed following sevoflurane treatment in U251 cells. The untreated control group acted as a negative control group for the experiments. Decreased VEGF expression was identified in the sevoflurane group compared with the control group, which was subsequently reversed following treatment with the miR-27b inhibitor (Fig. 4B). Moreover, the same results were obtained in U87 cells (Fig. 4C and D).

Sevoflurane-induced inhibition of glioma cell proliferation is mediated by the miR-27b/VEGF axis. To further assess the effects of sevoflurane on glioma cell proliferation, U251 and U87 cells were treated with miR-27b inhibitors or VEGF siRNA + miR-27b inhibitor prior to sevoflurane exposure.

The effect of VEGF siRNA on the expression of VEGF in U251 and U87 cells was determined via RT-qPCR. The present results suggested that, compared with the siRNA-NC group, VEGF siRNA-1 and VEGF siRNA-2 significantly decreased the expression of VEGF, with siRNA-2 demonstrating a more significant effect (Fig. 5A and B). Therefore, VEGF siRNA-2 was used for subsequent experimentation.

It was demonstrated that the inhibitory effects of sevoflurane on U251 and U87 cell proliferation were abolished by 
A

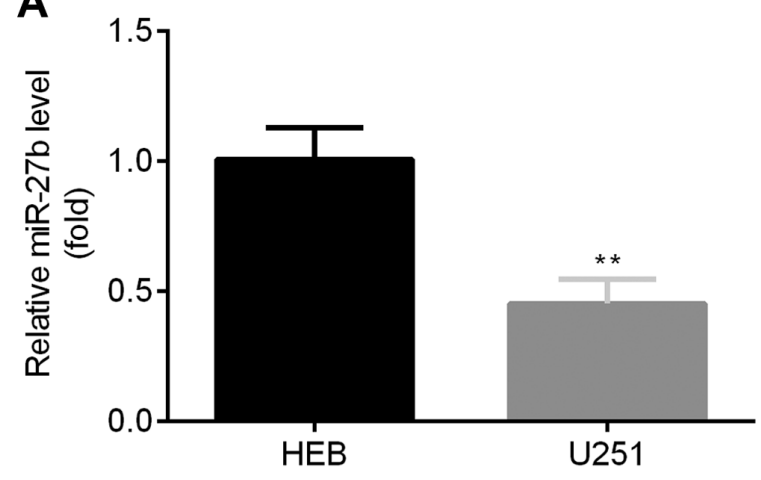

C

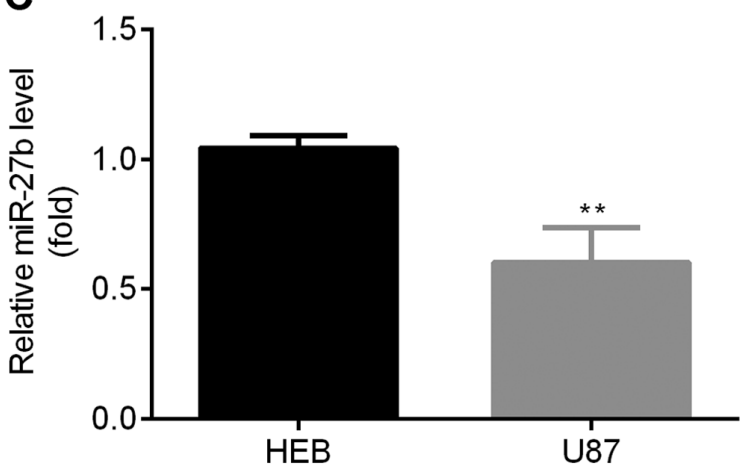

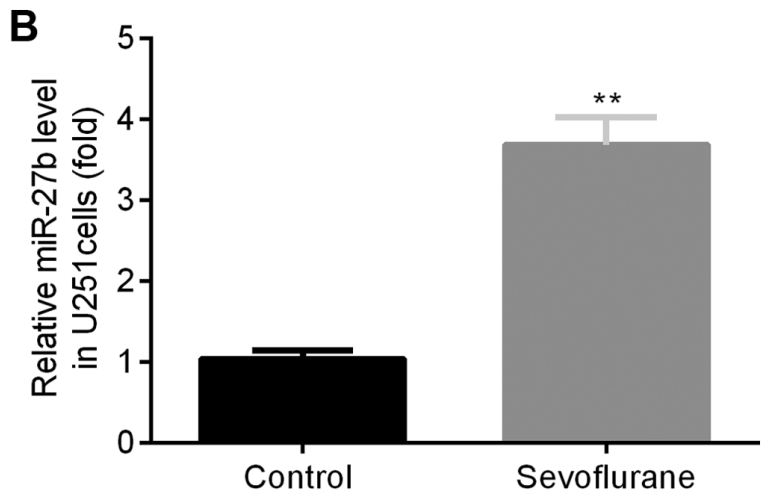

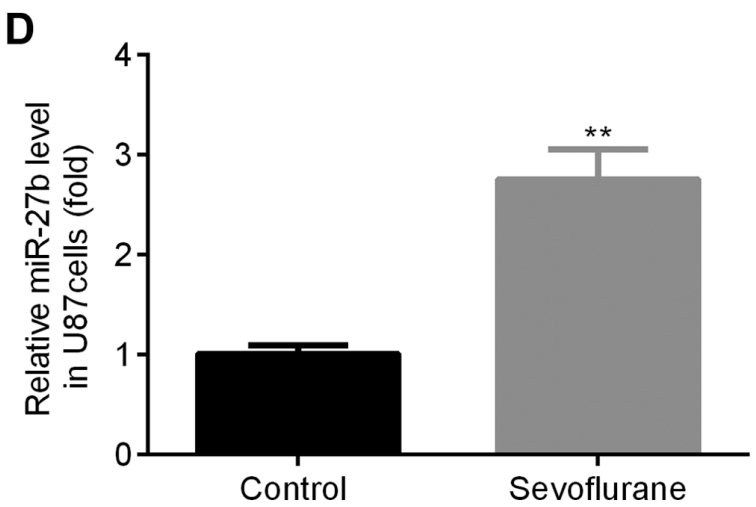

Figure 1. Sevoflurane induces the expression of miR-27b in glioma cells. Decreased miR-27b lower expression was identified in (A) U251 cells compared with HEB cells, which was significantly increased by (B) sevoflurane. Similar miR-27b expression levels were found in (C) U87 cells and (D) after sevoflurane treatment. ${ }^{* *} \mathrm{P}<0.01$ vs. HEB, or sevoflurane vs. control. miR, microRNA.
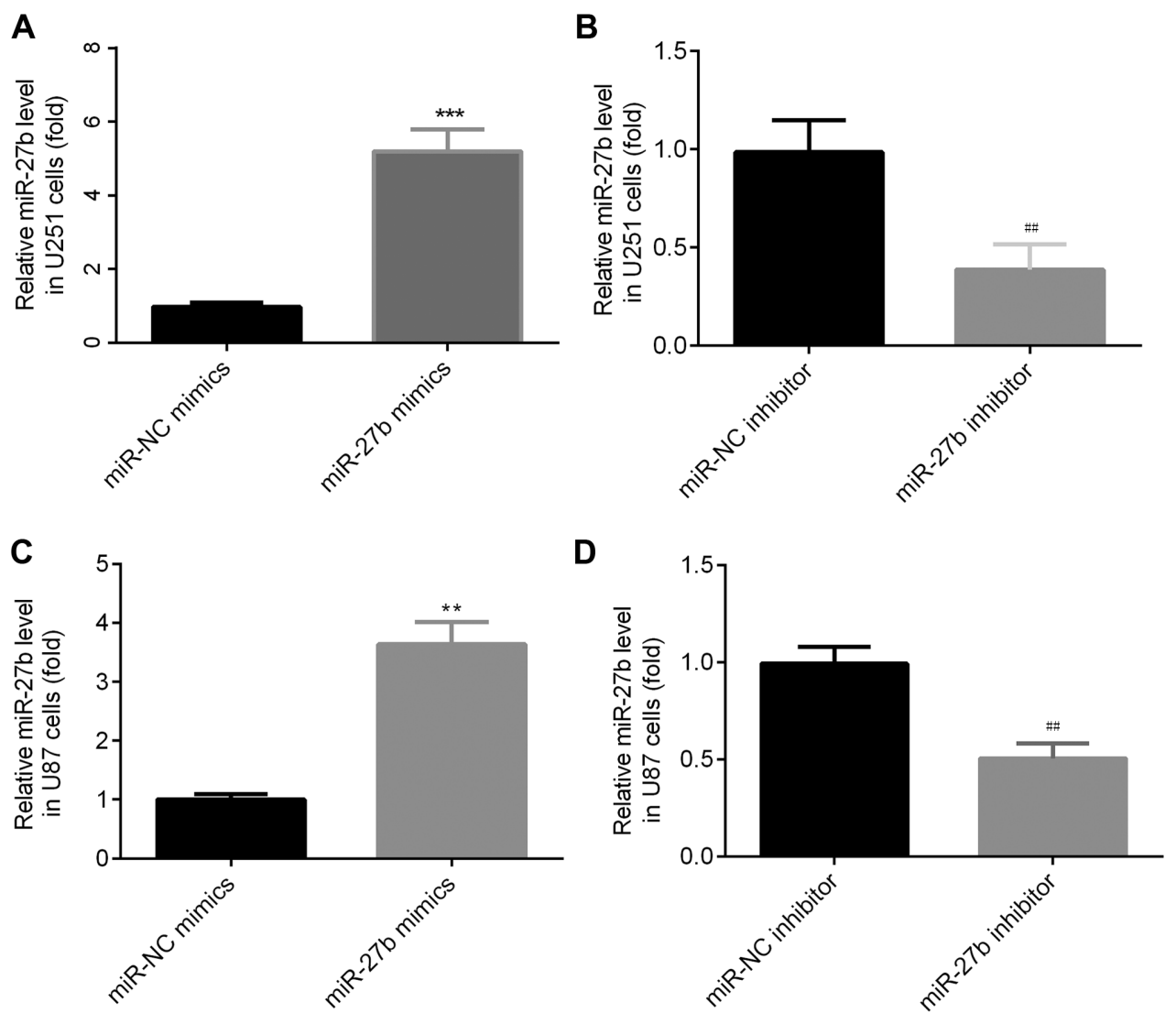

Figure 2. miR-27b mimic and inhibitor affect miR-27b expression levels. In (A) U251 cells, miR-27b was significantly increased after miR-27b mimics transfection, while significantly decreased after (B) miR-27b inhibitor transfection. Similar results were obtained in U87 cells after (C) miR-27b mimic and (D) inhibitor transfection. ${ }^{* *} \mathrm{P}<0.01,{ }^{* * * *} \mathrm{P}<0.001$, miR-27b mimics vs. miR-NC mimics; ${ }^{\# \#} \mathrm{P}<0.01$, miR-27b inhibitor vs. miR-NC inhibitor. miR, microRNA; NC, negative control. 
A

\section{5' ATTGGTAAAACTCTTCGTGAT 3' VEGF 3'UTR-Mutant 3' CGTCTTGAATCGGTGACACTT 5' hsa-miR-27b \\ 5' ATTGGTAAAACTCACTGTGAT 3' VEGF 3'UTR-WT}

B

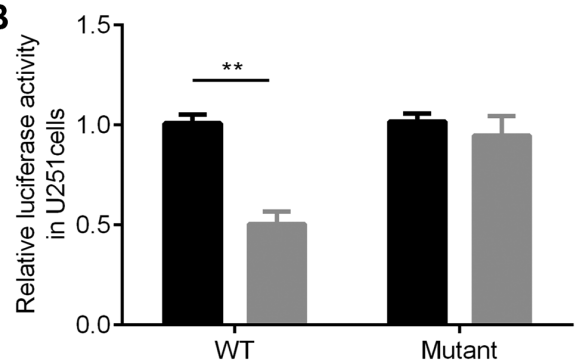

C

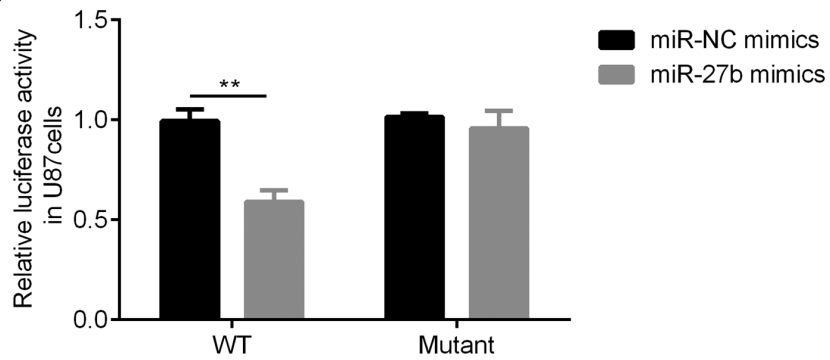

Figure 3. VEGF is a target of miR-27b. (A) A conserved binding site for miR-27b in the 3'UTR region of the VEGF gene was identified. miR-27b targeted VEGF 3'UTR in (B) U251 and (C) U87 cells. ${ }^{* *} \mathrm{P}<0.01 \mathrm{miR}-27 \mathrm{~b}$ mimics vs. miR-NC mimics. miR, microRNA; NC, negative control; 3'UTR, 3 untranslated region; WT, wild-type; VEGF, vascular epithelial growth factor.

pre-treatment with the miR-27b inhibitor (Fig. 5C and D). However, no effect was identified when cells were treated with VEGF siRNA + miR-27b inhibitor (Fig. 5C and D).

Sevoflurane-induced inhibition of glioma cell migration is mediated by the miR-27b/VEGF axis. To further investigate the effects of sevoflurane on glioma cell migration, U251 cells were treated with miR-27b inhibitor or siRNA VEGF + miR-27b inhibitor prior to sevoflurane exposure. It was found that the inhibitory effect of sevoflurane on U251 cell migration was partially reversed by the pre-treatment with the miR-27b inhibitor; however, no effect was observed when cells were treated with siRNA VEGF + miR-27b inhibitor (Fig. 6A and B). Furthermore, similar results were obtained in U87 cells (Fig. 6C and D). The present results indicated that there were no significant pathological morphology changes in U251 or U87 cells (Fig. 6A-D).

Sevoflurane inhibits the expression levels of MMP-2 and MMP-9. To determine whether sevoflurane inhibits the expression levels of MMP-2 and MMP-9, U251 and U87 cells were pre-treated with miR-27b inhibitors or VEGF siRNA + miR-27b inhibitors, and these proteins were assessed following sevoflurane exposure. The untreated control group acted as a negative control group for the experiments.

The present results suggested that the decreased protein expression levels of MMP-2 and MMP-9 induced by sevoflurane in U251 cells were reversed following pre-treatment with the miR-27b inhibitor. However, no effect was identified following VEGF siRNA + miR-27b inhibitor treatment compared with sevoflurane treatment (Fig. 7A and B). Moreover, similar effects were demonstrated in U87 cells (Fig. 7C and D).

\section{Discussion}

Sevoflurane has been shown to exert anti-proliferative effects in colon cancer (9) and laryngeal cancer cells (10). Furthermore, sevoflurane prevents the migration of lung cancer cells (11) and regulates various miRNAs of the brain (12). Moreover, sevoflurane was reported to inhibit the migration and invasion of glioma cells by upregulating miR-637 (22). Various miRNAs have also been revealed to inhibit glioma cell proliferation and migration, including miR-10b (23), miR-218 (24) and miR-29c (25). The inhibitory effects of miR-27b on the progression of cancer have been identified in different cancer types: miR-27b inhibits cell proliferation and migration by targeting PI3K p1 $10 \alpha$ in colorectal cancer (26), and long non-coding RNA DLX6-AS1 induces the progression of lung cancer by targeting miR-27b-3p (27). With regard to glioma, the role of miR-27b is controversial. It has been shown that miR-27b promotes cell invasion in glioma cells (28), and that upregulation of miR-27b contributes to the malignancy of glioma (29), which indicates that miR-27b has an oncogenic role. However, miR-27b expression is significantly lower in metastatic glioma tissues compared with non-metastatic tissues (20), and miR-27b is decreased in metastatic tumors of glioma (20), which indicates that miR-27b acts as a tumor suppressor. Yet, whether sevoflurane inhibits glioma cell migration and proliferation by regulating miR-27b is not fully understood.

The present results indicated that there were significantly decreased expression levels of miR-27b in U251 and U87 cells compared with HEB cells. Furthermore, sevoflurane treatment significantly increased miR-27b expression in cells compared with the control group. Thus, the present results suggested that miR-27b may serve as a tumor suppressor in glioma, which is consistent with previous studies (20). Furthermore, it was speculated that sevoflurane may serve a pivotal role in the progression of glioma by upregulating miR-27b.

miRNAs negatively regulate gene expression by inhibiting translation or inducing the degradation of target mRNA (30). Therefore, future studies should focus on mRNAs that function as oncogenes.

Using computational methods, the present study predicted that miR-27b targeted the 3'UTR of VEGF. Adequate blood supply is indispensable for tumor growth and metastasis. As neoplasms grow larger, adequate blood supply is obtained via angiogenesis (31). Moreover, tumor cells produce various inducers of angiogenesis, including VEGF, which has important roles in endothelial survival, proliferation and new vessel formation (32). To assess whether miR-27b targeted the 3'UTR of VEGF, a dual-luciferase reporter assay was performed. The present results indicated that there was increased VEGF expression in U251 and U87 cells compared with HEB cells. In addition, compared with the control group, decreased VEGF expression was demonstrated in the sevoflurane group, which was subsequently rescued following treatment with the miR-27b inhibitor.

The effects of miR-27b on the sevoflurane-induced proliferation and migration of U251 and U87 cells were examined 

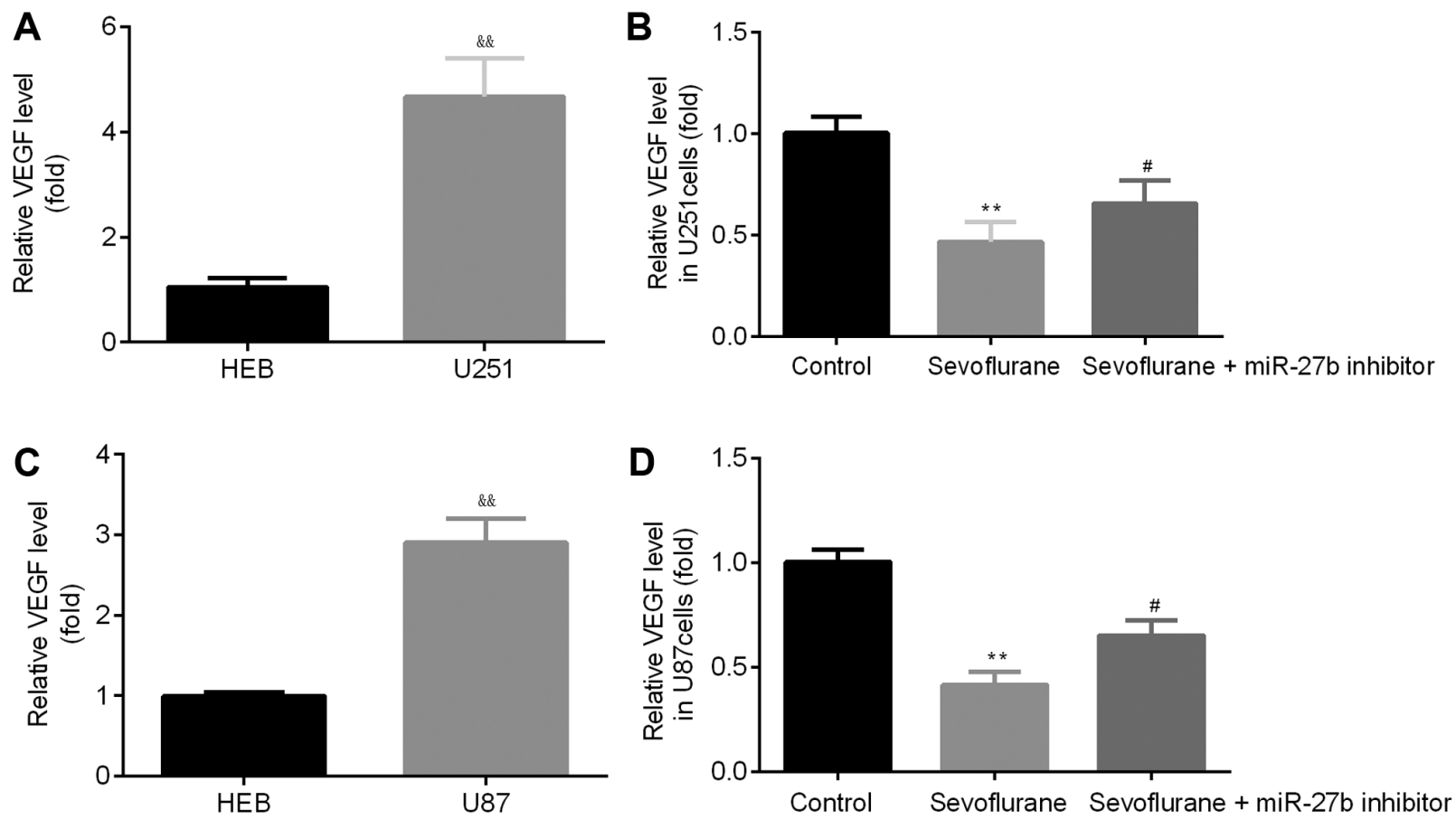

Figure 4. Sevoflurane reduces the expression of VEGF in glioma cells by targeting miR-27b. (A) Increased expression of VEGF in U251 cells compared with HEB cells. The untreated control group acted as a negative control group for the experiments. (B) Compared with control group, there was decreased VEGF expression in the sevoflurane group, which was rescued by miR-27b inhibitor. (C) Increased expression of VEGF in U87 cells compared with HEB cells. (D) Compared with the control group, there was decreased VEGF expression in the sevoflurane group, which was rescued by the miR-27b inhibitor. ${ }^{\& \&} \mathrm{P}<0.01$, U251/U87 vs. HEB. ${ }^{* *} \mathrm{P}<0.01$, sevoflurane vs. control. ${ }^{*} \mathrm{P}<0.05$ sevoflurane + miR-27b inhibitor vs. sevoflurane. miR, microRNA; VEGF, vascular epithelial growth factor.
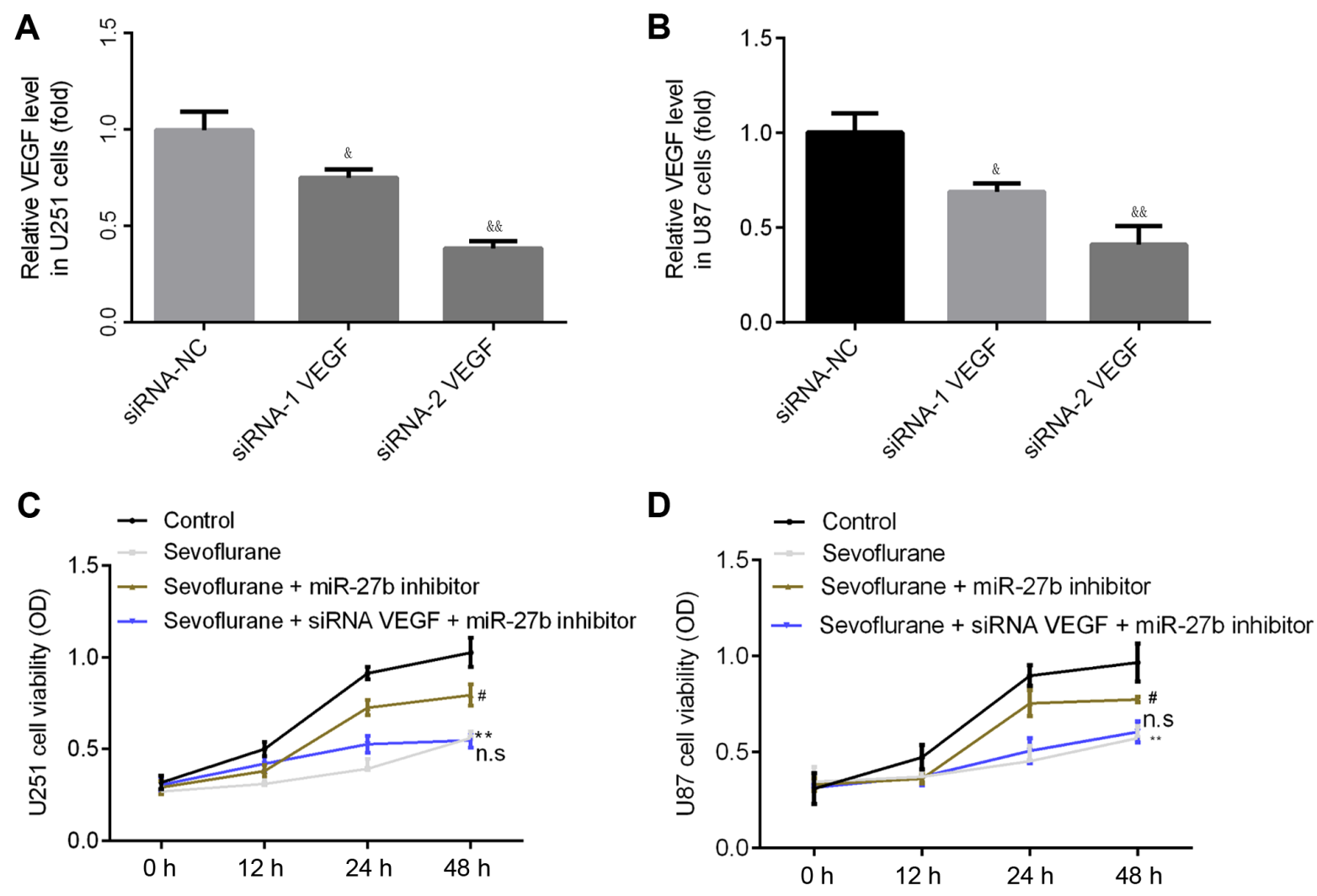

Figure 5. Sevoflurane-induced inhibition of glioma cell proliferation is mediated by the miR-27b/VEGF axis. Compared with the siRNA-NC group, siRNA-1 VEGF and siRNA-2 VEGF significantly decreased the expression of VEGF in (A) U251 and (B) U87 cells. The untreated control group acted as a negative control group for the experiments. The inhibitory effects of sevoflurane on (C) U251 and (D) U87 cell proliferation were abolished by pre-treatment with miRNA-27b inhibitor, but were not affected by siRNA VEGF + miR-27b inhibitor. ${ }^{\circledR} \mathrm{P}<0.05$ siRNA-1 VEGF vs. siRNA-NC. \&\&P<0.01 siRNA-2 VEGF vs. siRNA-NC. ${ }^{* *} \mathrm{P}<0.01$ sevoflurane vs. control. ${ }^{~} \mathrm{P}<0.05$ sevoflurane + miR-27b inhibitor vs. sevoflurane. $\mathrm{n} . \mathrm{s}$, sevoflurane + siRNA VEGF + miR-27b inhibitor vs. sevoflurane. n.s, not significant; siRNA, small interfering RNA; miR, microRNA; VEGF, vascular epithelial growth factor. 
A
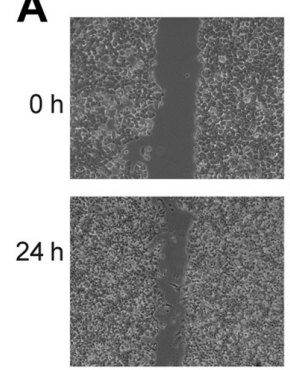

Sevoflurane miR 27b inhibitor SiRNA VEGF

C
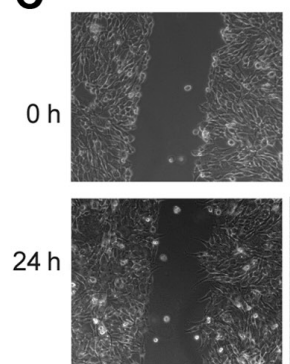

Sevoflurane miR 27b inhibitor SIRNA VEGF
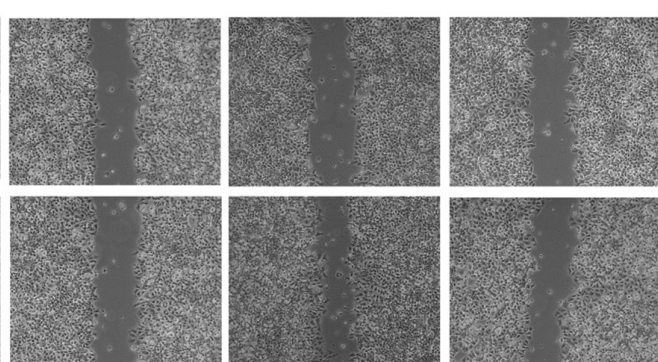

$+$
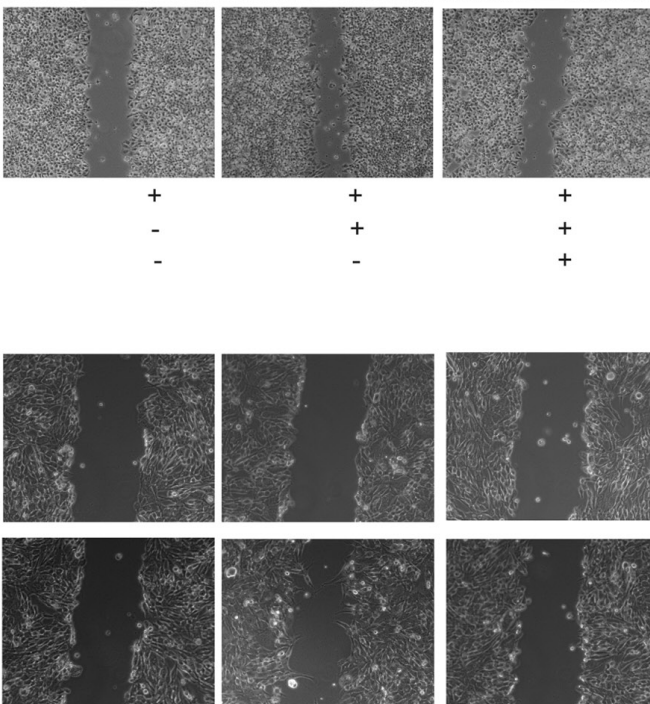

$+$

$-$

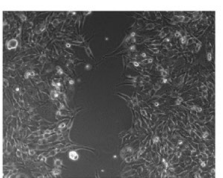

$+$

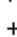

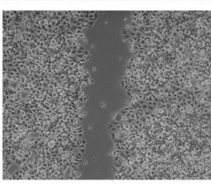

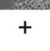

$+$

B

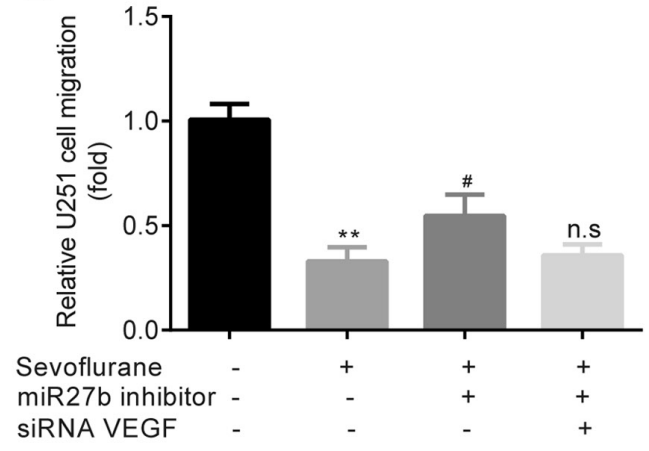

D

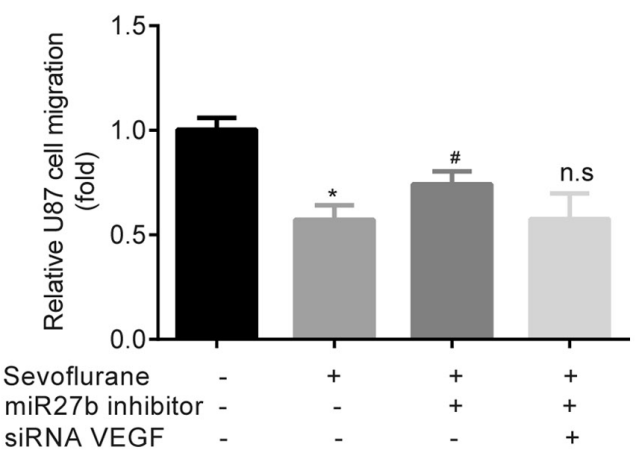

Figure 6. Sevoflurane-induced inhibition of glioma cell migration is mediated by the miR-27b/VEGF axis. The untreated control group acted as a negative control group for the experiments. (A) Images and (B) analysis of the inhibitory effects of sevoflurane on U251 cell migration were abolished by pre-treatment with miR-27b inhibitor, but were not affected by siRNA VEGF + miR-27b inhibitor. (C) Images and (D) analysis of the inhibitory effects of sevoflurane on U87 cell migration were abolished by pre-treatment with miR-27b inhibitor, but were not affected by siRNA VEGF + miR-27b inhibitor. Magnification, $x 100$. ${ }^{*} \mathrm{P}<0.05$ and ${ }^{* *} \mathrm{P}<0.01$ sevoflurane vs. control. ${ }^{*} \mathrm{P}<0.05$ sevoflurane + miR-27b inhibitor vs. sevoflurane. n.s, sevoflurane + siRNA VEGF + miR-27b inhibitor vs. sevoflurane. n.s, not significant; siRNA, small interfering RNA; miR, microRNA; VEGF, vascular epithelial growth factor.

A

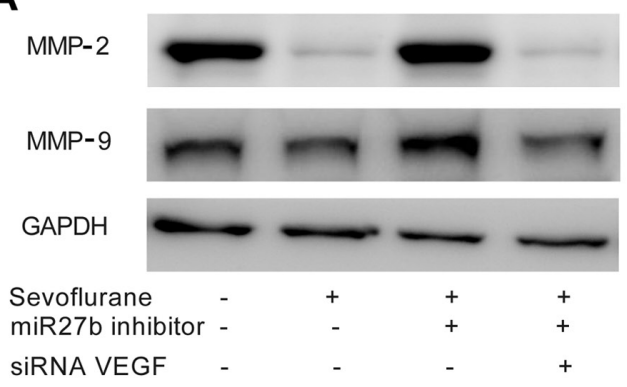

C

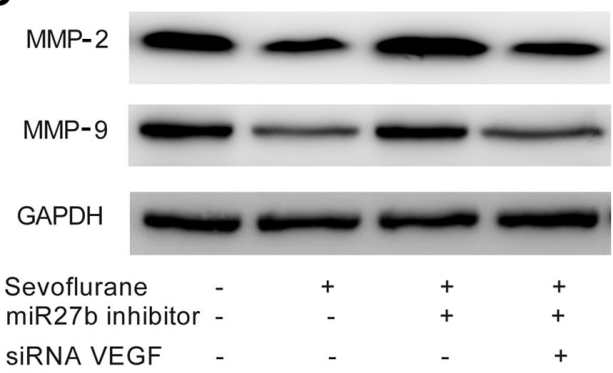

B

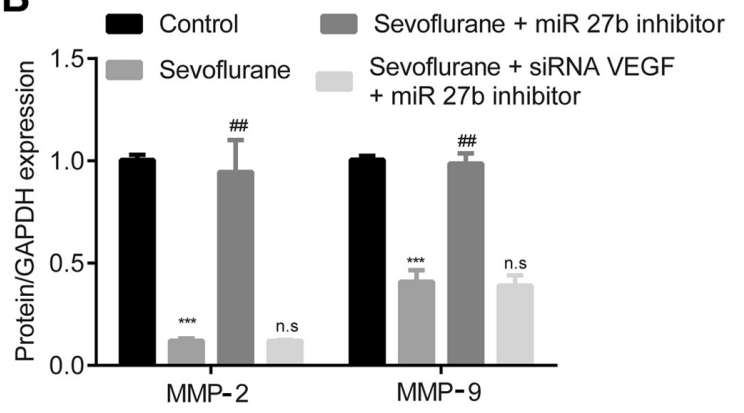

D

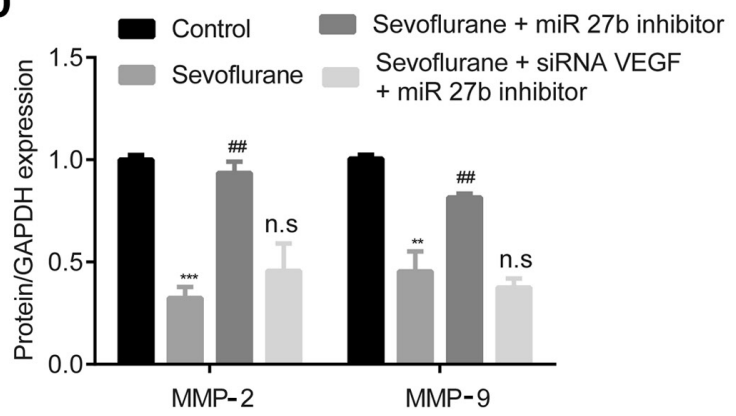

Figure 7. Sevoflurane inhibits the expression levels of MMP-2 and MMP-9. (A) Decreased in protein expression levels of MMP-2 and MMP-9 in U251 cells induced by sevoflurane were reversed by pre-treatment with miR-27b inhibitor. However, these were not affected by siRNA VEGF + miR-27b inhibitor. (B) Quantification of western blotting results. (C) Decreased in protein expression levels of MMP-2 and MMP-9 in U87 cells induced by sevoflurane were reversed by pre-treatment with miR-27b inhibitor. However, these were not affected by siRNA VEGF + miR-27b inhibitor. (D) Quantification of western blotting results. ${ }^{* *} \mathrm{P}<0.01$ and ${ }^{* * *} \mathrm{P}<0.001$ sevoflurane vs. control. ${ }^{\# \#} \mathrm{P}<0.01$ sevoflurane + miR-27b inhibitor vs. sevoflurane. n.s, sevoflurane + siRNA VEGF + miR-27b inhibitor vs. sevoflurane. n.s, not significant; siRNA, small interfering RNA; miR, microRNA; VEGF, vascular epithelial growth factor; MMP, matrix metalloproteinase. 
in the present study. It was found that the inhibitory effects of sevoflurane on U251 and U87 cell proliferation and migration were abolished following pre-treatment with the miR-27b inhibitor. However, no effects were identified following treatment with VEGF siRNA + miR-27b inhibitor.

MMPs are a family of zinc-dependent endopeptidases that degrade components of the extracellular matrix (ECM). Furthermore, MMP activation induces cell migration and invasion (33). MMP-2 is a key enzyme that degrades certain components of the ECM, including type IV (34). Moreover, the present results suggested that the sevoflurane-induced decrease of MMP-2 and MMP-9 protein expression levels in U251 and U87 cells was reversed following pre-treatment with the miR-27b inhibitor. However, no effects were demonstrated following treatment with VEGF siRNA + miR-27b inhibitor.

However, there are several limitations in the present study. Firstly, there are several mRNAs that could be targeted by miR-27b and the present study only investigated the role of VEGF. Thus, future studies will investigate these other potential targets. Secondly, the culture medium for the wound healing assay contained $1 \%$ FBS, which could influence the migration, therefore it will be replaced by serum-free culture medium in the future.

In conclusion, the present results indicated that $\mathrm{miR}-27 \mathrm{~b}$ may serve as a tumor suppressor and that VEGF acts as an oncogene in glioma. Therefore, miR-27b may be a novel target for sevoflurane in glioma. Moreover, it was found that sevoflurane inhibited glioma cell proliferation and migration, which may be related to miR-27b and its target VEGF. Collectively, the present results indicated that targeting the miR-27b/VEGF axis may be a potential treatment for glioma.

\section{Acknowledgements}

Not applicable.

\section{Funding}

No funding was received.

\section{Availability of data and materials}

The datasets used and/or analyzed during the current study are available from the corresponding author on reasonable request.

\section{Authors' contributions}

$\mathrm{XZ}$ and CL performed the experiments and analyzed the data. LY conceived the study, analyzed the data and prepared the manuscript. All authors read and approved the final manuscript.

\section{Ethics approval and consent to participate}

Not applicable.

\section{Patient consent for publication}

Not applicable.

\section{Competing interests}

The authors declare that they have no competing interests.

\section{References}

1. Mamelak AN and Jacoby DB: Targeted delivery of antitumoral therapy to Glioma and other malignancies with synthetic chlorotoxin (TM-601). Expert Opin Drug Deliv 4: 175-186, 2007.

2. Radner H, El-Shabrawi Y, Eibl RH, Brüstle O, Kenner L, Kleihues $\mathrm{P}$ and Wiestler OD: Tumor induction by ras and myc oncogenes in fetal and neonatal brain: Modulating effects of developmental stage and retroviral dose. Acta Neuropathol 86: 456-465, 1993.

3. Wong ET and Brem S: Taming glioblastoma: Targeting angiogenesis. J Clin Oncol 25: 4705-4706, 2007.

4. Jiang B, Chaichana K, Veeravagu A, Chang SD, Black KL and Patil CG: Biopsy versus resection for the management of low-grade gliomas. The Cochrane Database of Systematic Reviews 4: CD009319, 2017.

5. Stewart L, Burdett S and Glioma Meta-analysis Trialists Group (GMT): Chemotherapy for high-grade glioma. Cochrane Database Syst Rev: CD003913, 2012.

6. Davis FG and McCarthy BJ: Current epidemiological trends and surveillance issues in brain tumors. Expert Rev Anticancer Ther 1: 395-401, 2001

7. Giese A, Bjerkvig R, Berens ME and Westphal M: Cost of migration: Invasion of malignant gliomas and implications for treatment. J Clin Oncol 21:1624-1636, 2003.

8. Snyder GL and Greenberg S: Effect of anaesthetic technique and other perioperative factors on cancer recurrence. Br J Anaesth 105: 106-115, 2010.

9. Kvolik S, Glavas-Obrovac L, Bares V and Karner I: Effects of inhalation anesthetics halothane, sevoflurane, and isoflurane on human cell lines. Life Sci 77: 2369-2383, 2005.

10. Kvolik S, Dobrosevic B, Marczi S, Prlic L and Glavas-Obrovac L: Different apoptosis ratios and gene expressions in two human cell lines after sevoflurane anaesthesia. Acta Anaesthesiol Scand 53: 1192-1199, 2009.

11. Liang $\mathrm{H}, \mathrm{Gu} M$, Yang $\mathrm{C}$, Wang $\mathrm{H}$, Wen $\mathrm{X}$ and Zhou Q: Sevoflurane inhibits invasion and migration of lung cancer cells by inactivating the p38 MAPK signaling pathway. J Anesth 26: 381-392, 2012.

12. Goto G, Hori Y, Ishikawa M, Tanaka S and Sakamoto A: Changes in the gene expression levels of microRNAs in the rat hippocampus by sevoflurane and propofol anesthesia. Mol Med Rep 9: 1715-1722, 2014.

13. Ambros V: microRNAs: Tiny regulators with great potential. Cell 107: 823-826, 2001.

14. Bartel DP: MicroRNAs: Genomics, biogenesis, mechanism, and function. Cell 116: 281-297, 2004.

15. Kloosterman WP and Plasterk RH: The diverse functions of microRNAs in animal development and disease. Dev Cell 11: 441-450, 2006.

16. Ciafre SA, Galardi S, Mangiola A, Ferracin M, Liu CG, Sabatino G, Negrini M, Maira G, Croce CM and Farace MG: Extensive modulation of a set of microRNAs in primary glioblastoma. Biochem Biophys Res Commun 334: 1351-1358, 2005.

17. Feng Q, Wu X, Li F, Ning B, Lu X, Zhang Y, Pan Y and Guan W: miR-27b inhibits gastric cancer metastasis by targeting NR2F2 . Protein Cell 8: 114-122, 2017.

18. Sun Y, Xu T, Cao YW and Ding XQ: Antitumor effect of miR-27b-3p on lung cancer cells via targeting Fzd7. Eur Rev Med Pharmacol Sci 21: 4113-4123, 2017.

19. Luo Y, Yu SY, Chen JJ, Qin J, Qiu YE, Zhong M and Chen M: MiR-27b directly targets Rab3D to inhibit the malignant phenotype in colorectal cancer. Oncotarget 9: 3830-3841, 2017.

20. Khan FH, Pandian V, Ramraj S, Aravindan S, Herman TS and Aravindan N: Reorganization of metastamiRs in the evolution of metastatic aggressive neuroblastoma cells. BMC Genomics 16: $501,2015$.

21. Livak KJ and Schmittgen TD: Analysis of relative gene expression data using real-time quantitative PCR and the 2(-Delta Delta C(T)) method. Methods 25: 402-408, 2001.

22. Yi W, Li D, Guo Y, Zhang Y, Huang B and Li X: Sevoflurane inhibits the migration and invasion of glioma cells by upregulating microRNA-637. Int J Mol Med 38: 1857-1863, 2016. 
23. Lin J, Teo S, Lam DH, Jeyaseelan K and Wang S: MicroRNA-10b pleiotropically regulates invasion, angiogenicity and apoptosis of tumor cells resembling mesenchymal subtype of glioblastoma multiforme. Cell Death Dis 3: e398, 2012.

24. Song L, Huang Q, Chen K, Liu L, Lin C, Dai T, Yu C, Wu Z and Li J: miR-218 inhibits the invasive ability of glioma cells by direct downregulation of IKK- $\beta$. Biochem Biophys Res Commun 402: 135-140, 2010.

25. Fan YC, Mei PJ, Chen C, Miao FA, Zhang H and Li ZL: MiR-29c inhibits glioma cell proliferation, migration, invasion and angiogenesis. J Neurooncol 115: 179-188, 2013.

26. Chen Y, Zhang B, Jin Y, Wu Q and Cao L: MiR-27b targets PI3K $\mathrm{p} 110 \alpha$ to inhibit proliferation and migration in colorectal cancer stem cell. Am J Transl Res 11: 5988-5997, 2019.

27. Sun W, Zhang L, Yan R, Yang Y and Meng X: LncRNA DLX6-AS1 promotes the proliferation, invasion, and migration of non-small cell lung cancer cells by targeting the miR-27b-3p/GSPT1 axis Onco Targets Ther 12: 3945-3954, 2019.

28. Liu C, Liang S, Xiao S, Lin Q, Chen X, Wu Y and Fu J: MicroRNA-27b inhibits Spry2 expression and promotes cell invasion in glioma U251 cells. Oncol Lett 9: 1393-1397, 2015.

29. Chen L, Li H, Han L, Zhang K, Wang G, Wang Y, Liu Y, Zheng Y, Jiang T, Pu P, et al: Expression and function of miR-27b in human glioma. Oncol Rep 26: 1617-1621, 2011.
30. Wen MM: Getting miRNA therapeutics into the target cells for neurodegenerative diseases: A mini-review. Front Mol Neurosci 9: 129, 2016

31. Dhup S, Dadhich RK, Porporato PE and Sonveaux P: Multiple biological activities of lactic acid in cancer: Influences on tumor growth, angiogenesis and metastasis. Curr Pharm Des 18: 1319-1330, 2012

32. Yancopoulos GD: Clinical application of therapies targeting VEGF. Cell 143: 13-16, 2010.

33. Stetler-Stevenson WG and Seo DW: TIMP-2: An endogenous inhibitor of angiogenesis. Trends $\mathrm{M}$ ol Med 11: 97-103, 2005.

34. Tapia A, Salamonsen LA, Manuelpillai U and Dimitriadis E: Leukemia inhibitory factor promotes human first trimester extravillous trophoblast adhesion to extracellular matrix and secretion of tissue inhibitor of metalloproteinases-1 and -2. Hum Reprod 23: 1724-1732, 2008.

This work is licensed under a Creative Commons Attribution-NonCommercial-NoDerivatives 4.0 International (CC BY-NC-ND 4.0) License. 